ISSN: 1808-8759

\title{
ALTERAÇÕES NUTRICIONAIS EM CASCA E POLPA DE CENOURA DECORRENTE DE DIFERENTES MÉTODOS DE COZIMENTO
}

\author{
Daniela Regina Pigoli ${ }^{1}$, Rogério Lopes Vieites ${ }^{2}$ \& Érica Regina Daiuto ${ }^{3}$
}

\begin{abstract}
RESUMO: O objetivo desse trabalho foi verificar o método de cozimento mais adequado para o preparo da cenoura (cv Nantes), visando minimizar as perdas nutricionais. As cascas e as fatias de cenoura foram submetidas a pré testes de cozimento a fim de se determinar o melhor tempo de cozimento, iniciando-se com 0,5 min, $1 \mathrm{~min}, 1,5 \mathrm{~min}$ e sucessivamente, até alcançar o grau de maciez que foi avaliado subjetivamente por pressão das hortaliças entre os dedos, alcançando uma consistência adequada ao consumo. As cascas e as fatias de cenouras foram submetidas a quatro tipos de tratamentos térmicos (pressão, imersão, microondas e vapor), após, foram trituradas em mix doméstico e armazenadas à $-18^{\circ} \mathrm{C}$. A avaliação das perdas nutricionais da hortaliça foi feita pela determinação de proteínas, lipídios, fibras, teores de açúcares redutores, totais assim como de ácido ascórbico e minerais (ferro, zinco, magnésio, potássio, fósforo e cálcio). As análises foram realizadas na hortaliça fresca e após cozimento pelos diferentes métodos em estudo. A casca da cenoura apresentou porcentagens de proteínas, lipídios, fibras, teores de açúcares redutores, totais assim como de ácido ascórbico equivalente a polpa. Já o conteúdo de minerais foi superior na casca da cenoura em relação à polpa. As porcentagens foram respectivamente de 38,10\%, 95,12\%, 47,04\%, 58,88\%, 70,27\% e 21,27\% a mais em relação à polpa podendo portanto, ser aproveitada no preparo de refeições. Ocorreram perdas de nutrientes na polpa e casca de cenoura submetidas aos diferentes métodos de cozimento em relação ao vegetal cru. O método de cozimento à vapor e em microondas resultaram nas menores perdas nutricionais na polpa e casca de cenoura.
\end{abstract}

PALAVRAS-CHAVE: Daucus carota, vitamina C, processamento.

\section{NUTRITIONAL ALTERATIONS IN CARROT PEEL AND PULP DUE TO DIFFERENT COOKING METHODS}

ABSTRACT: The aim of this study was to verify the carrot cooking most suitable method to minimize nutrient losses. Carrot peel slices were subjected to pre cooking tests that were initiated with 0.5 min of duration and then increased in 0.5 min successively. The carrot pieces texture was monitored during the pre tests so all would havethe same texture independent of the type of cooking. The degree of softennes was evaluated by pressuring the pieces between the toes. The carrot pulp and pell were subjected to four types of heat treatment (pressure, immersion, microwave, and steam), after that they were pounded with a food processor and stored at $-18{ }^{\circ} \mathrm{C}$. The nutritional analyses were as follow: The evalu determination of proteins, lipids, fibers, sugars reducers, total of ascorbic acid content and minerals (iron, calcium, zinc, magnesium, potassium, phosphorus, and calcium). The analyses were accomplished with fresh carrot and after cooking with the different methods. The peel of the carrot presented as amounts of proteins, lipids, fibers percentages, sugars reducers, total and ascorbic acid content equivalent to the pulp. In addition, the minerals content was superior in the peel in relation to the pulp, presenting respective percentages of 38,10\%, 95,12\%, 47,04\%, 58,88\%, 70,27\% and $21,27 \%$. There were nutrient losses in relation to the raw vegetable, when the carrot pieces were submitted to the different cooking methods. The methods of steaming and microwave had lower nutritional losses .

KEYWORDS: Daucus carota, vitamin C, processing.

\section{INTRODUÇÃO}

A cenoura é uma das hortaliças mais produzidas, sendo de grande emprego na indústria de alimentos. Pertencente ao

\footnotetext{
${ }^{1}$ E-mail: danipigoli@ig.com.br

${ }^{2}$ E-mail: vieites@fca.unesp.br

${ }^{3}$ E-mail: erdaiuto@uol.com.br
}

grupo das raízes tuberosas sendo cultivada em larga escala nas regiões Sudeste, Nordeste e Sul do Brasil, e está entre os cincos principais produtos hortalícios cultivados no Brasil. Os principais produtores são Minas Gerais, São Paulo, Paraná e Bahia e sua produção chega a 25 mil toneladas (AGROOLHAR, 2013). Pode ser processada para conserva enlatada, em mistura com outras hortaliças ou também na forma 
desidratada (SPAGNOLl, PARK; SIGRIST, 2006). Outra forma de comercialização é como produto minimamente processado, podendo ser encontrada ralada em secções de diversos tamanhos, ser picada na forma de fatias, cubos e palitos ou ainda como mini-cenoura (LANA, 2000). Lima et al. (2004) citam as linhas infantis na forma de conservas apertizadas, além de produção de picles, congeladas, desidratadas e sucos.

O consumo da cenoura, assim como de outras frutas e hortaliças, está associado a uma dieta saudável e a prevenção de doenças. A inclusão de hortaliças variadas na alimentação cotidiana é benéfica devido ao efeito alcalinizante sistêmico desses vegetais, que além de favorecer o preenchimento das cotas de vitaminas e minerais, aumentam a formação dos resíduos alimentares no trato gastrintestinal (FRANCO, 2004).

O componente em maior abundância na cenoura é a água (88,1-91,9\%). As restantes substâncias são praticamente: açúcares simples e complexos (glucose, frutose, sacarose, amido, substâncias pécticas e celulose), vitaminas, ácidos orgânicos, azoto e compostos minerais, lipídios e taninos (ZADERNOWSKI et al., 2003).

As cenouras são as principais fontes de origem vegetal em carotenóides provitamínicos A, especialmente o $\alpha$ e $\beta$ caroteno, e podem ser transformados em vitamina A no organismo animal. De acordo com Ramos (1991), os principais carotenóides em cenouras cruas da variedade Nantes são o $\beta$-caroteno (51,3\%), o $\alpha$-caroteno (29,5\%) e o $\gamma$-caroteno $(5,1 \%)$

As perdas de nutrientes em vegetais ocorrem quando acontece algum tipo de processamento: métodos de cocção, congelamento, pré-preparo, secagem ou processamento mínimo. Conforme Watada e Qi (1999) essas perdas são resultantes de injúrias nos tecidos vegetais, como descascamento, corte e centrifugação, normalmente utilizadas durante o processamento mínimo, provocando uma série de injúrias nos tecidos.

Muitas vezes, as hortaliças são consumidas na forma crua, mas há situações em que a cocção é necessária ou ainda preferida. Neste caso, o conteúdo e a capacidade dos nutrientes desses vegetais podem ser alterados, com consequências positivas e negativas, tal como a melhora da capacidade de compostos naturalmente presentes, a formação de novos compostos com atividade nutricional, a perda de nutrientes naturalmente presentes (CAMPOS et al., 2008).

A vitamina C é uma das vitaminas mais sensíveis a perdas em alimentos. Essa vitamina, na forma de ácido ascórbico, é muito susceptível à oxidação química e enzimática (RIOS; PENTEADO, 2003). A oxidação do ácido ascórbico ocorre especialmente na presença de oxigênio, íons metálicos, $\mathrm{pH}$ alcalino e temperaturas elevadas (COMBS, 1998). Além disso, a perda de vitamina C também pode se dar por lixiviação durante etapas de higienização dos alimentos, devido ao contato direto com a água (LEE; KADER, 2000).

Os minerais presentes nos alimentos também não são estáveis ao calor. Desse modo, as perdas desses nutrientes encontrados nos alimentos ocorrem de forma predominante durante as operações de descascamento, lavagem, corte e cozimentos. Pode ocorrer também a diminuição na disponibilidade de alguns íons como cálcio, ferro e zinco e pela interação com outros componentes dos alimentos como por exemplo: oxalatos, fitatos e taninos (SANTOS et al., 2003).

Portanto, a manutenção das quantidades de minerais e vitaminas nos vegetais é um desafio, pois, logo após a colheita já se iniciam reações químicas e físicas que alteram sua qualidade, sendo os principais determinadores das perdas os métodos de cocção, pois a temperatura, o tempo e o tipo de cocção influenciam diretamente na quantidade final destes nutrientes.

Além das perdas nutricionais observadas referentes aos métodos de processamentos existentes, observa-se o desperdício de hortaliças diariamente, principalmente de seus resíduos constituídos por partes denominadas não comestíveis.

No preparo doméstico da cenoura, a casca é descartada. Segundo Monteiro (2009), as partes não convencionais dos vegetais apresentam teores de ferro, vitamina C, cálcio e potássio próximos ou superiores às suas partes convencionais. Essas partes podem ser consideradas como fontes alternativas de nutrientes, ora auxiliando no alcance das necessidades nutricionais, assim como suas partes convencionais, ora colaborando para a diminuição do desperdício alimentar, contribuindo com a melhora do estado de saúde e qualidade de vida dos indivíduos.

Portanto, o objetivo desse trabalho foi verificar o método de cozimento mais adequado para o preparo de partes convencionais e não convencionais de cenouras, visando assim minimizar as perdas nutricionais.

\section{MATERIAL E MÉTODOS}

Utilizou-se raízes de cenouras, cultivar Nantes, obtidas no comércio da cidade de Botucatu.

Para o pré-preparo das amostras, as cenouras foram inicialmente lavadas em água corrente, logo após foram separadas em cascas e polpas, picadas com o auxilio de uma faca, e com o auxilio de um processador de alimentos industrial. O material foi dividido em cinco amostras, sendo quatro submetidas a métodos de cocção (vapor, imersão, microondas e pressão) e uma amostra in natura.

\subsection{Determinação de tempos de cozimento}

Após o pré-preparo, as amostras foram submetidas a prétestes de cozimentos a fim de determinar o melhor tempo para a cenoura. Os pré-testes iniciaram-se com 0,5 minuto, 1 minuto e terminou com o melhor tempo e a consistência.

Para os procedimentos de cozimento, pesou-se 200 gramas de casca e polpa de cenouras, para cada tipo de cozimento e para cada repetição. Para todos os cozimentos, exceto o microondas, foi utilizado 1 litro de água em ebulição; no cozimento em imersão e na pressão, 
a amostra só foi adicionada à água após entrar em ebulição; no cozimento a vapor, a amostra também só foi adicionada ao equipamento após a fervura da água; no microondas o alimento foi adicionado em recipiente adequado sem água.

O critério adotado para escolha do melhor tempo de cozimento baseou-se na textura obtida. Para que todas as espécies obtivessem a mesma textura independente do tipo de cozimento, o grau de amolecimento foi avaliado subjetivamente por pressão das hortaliças entre os dedos, conforme recomendado por Ramirez-Cardenas, Leonel e Costa (2008), alcançando uma consistência branda.

\subsection{Preparo das amostras para análises}

Após o cozimento, as amostras foram trituradas em um mix, homogeneizadas e posteriormente acondicionadas em recipientes plásticos com tampa. A seguir, foram armazenadas sob congelamento lento a $-18{ }^{\circ} \mathrm{C}$, em recipientes plásticos para posterior análises.

\subsection{Métodos de cocção}

As cascas e rodelas de cenoura foram submetidas a quatro métodos de cocção (microondas, vapor, imersão e pressão) após determinados os tempos dos pré-testes, como descritos no item 2.1 (Tabela 1).

Tabela 1 - Métodos e tempos de cozimento utilizado para casca e polpa de cenoura.

\begin{tabular}{cccc}
\hline Tipo de cozimento & Polpa & Tempo de cozimento \\
\hline Imersão & $4 \mathrm{~min}$ & $2 \mathrm{~min}$ \\
Microondas & $3 \mathrm{~min}$ & $2 \mathrm{~min}$ \\
Pressão & & $30 \mathrm{~s}$ \\
Vapor & $6 \mathrm{~min}$ & \\
\hline
\end{tabular}

\subsection{Avaliação das alterações de nutrientes}

A avaliação das alterações de nutrientes deu-se a partir da análise nas amostras cruas e submetidas aos quatro tipos de cozimento. As análises realizadas foram:

- $\quad$ Proteína: determinada pelo método de Kjeldahl, modificado. Os valores para proteína foram calculados a partir do teor de nitrogênio total, utilizando o fator de conversão de 6,25 conforme descritos em AOAC (1984);

- Lipídios: realizada em extrator Soxhlet, utilizando éter de petróleo para a extração, conforme descritos em AOAC, (1984).

- Fibras: realizado pelo Método Henneberg, conforme descritos em (CECCHI, 1999).

- $\quad$ Açúcares redutores e totais: determinado pelo método de Somogy (1945), adaptado por Nelson (1944).

- $\quad$ Teor de ácido ascórbico: foi realizada por titulometria, baseando-se na redução do corante 2,6 diclofenol-indofenol pelo ácido ascórbico - Método Titulométrico (MAPA, 2001).

- $\quad$ Minerais: os elementos Fe, Ca, K, P, Mg e Zn, foi feita por Espectrofotometria de Absorção Atômica, segundo metodologia estabelecida por (MALAVOLTA; VITTI; OLIVEIRA, et al., 1989).
Todas as análises foram realizadas em triplicatas.

\subsection{Análise dos dados}

Foi realizada a análise de variância no delineamento inteiramente ao acaso, seguida do teste de Tukey para comparação de médias, ao nível de 5\% significância, utilizando-se o programa estatístico SAS.

\section{RESULTADOS E DISCUSSÃO}

Os teores de nutrientes no vegetal cru foram próximos para casca e polpa de cenoura, demonstrando que a parte não convencional apresenta valor nutricional idêntico à parte normalmente consumida (Tabela 2). Todos os nutrientes avaliados decresceram nas amostras submetidas aos diferentes tipos de cozimento em relação ao vegetal cru, tanto na polpa como na casca da cenoura. Gokoglu, Yerlikaya e Cengiz (2004) verificaram que, o cozimento pode alterar os valores de umidade, proteína, gordura e cinza dos alimentos em decorrência da incorporação do meio de cocção e das perdas de nutrientes na água. 
Tabela 2 - Teores de proteínas, lipídeos, fibras, açúcares e ácido ascórbico para a polpa e casca da cenoura, submetidas a diferentes métodos de cocção.

\begin{tabular}{|c|c|c|c|c|c|}
\hline $\begin{array}{ll}\text { Tipo } & \text { de } \\
\text { cozimento }\end{array}$ & Proteínas (\%) & Lipídeos (\%) & Fibras (\%) & $\begin{array}{c}\text { Açúcar } \\
\text { redutor(\%) }\end{array}$ & $\begin{array}{l}\text { Teor de ácido } \\
\text { ascórbico }\end{array}$ \\
\hline \multicolumn{6}{|c|}{ Polpa } \\
\hline Cru & $1,115 \mathrm{~A} \pm 0,069$ & $0,291 \mathrm{~A} \pm 0,030$ & $3,327 \mathrm{~A} \pm 0,297$ & $2,487 \mathrm{~A} \pm 0,168$ & $2,917 \mathrm{~A} \pm 0,144$ \\
\hline Imersão & $0,462 \mathrm{C} \pm 0,054$ & $0,126 \mathrm{~B} \pm 0,047$ & $1,306 \mathrm{~B} \pm 0,324$ & $0,515 \mathrm{~B} \pm 0,416$ & $1,078 \mathrm{~B} \pm 0,148$ \\
\hline \multirow[t]{2}{*}{ Vapor } & $0,644 \mathrm{~B} \pm 0,056$ & $0,145 \mathrm{~B} \pm 0,047$ & $1,598 \mathrm{~B} \pm 0,141$ & $0,691 \mathrm{~B} \pm 0,050$ & $1,332 \mathrm{BC} \pm 0,241$ \\
\hline & $0,486 \mathrm{C} \pm 0,063$ & $0,142 \mathrm{~B} \pm 0,041$ & $1,558 \mathrm{~B} \pm 0,139$ & $0,516 \mathrm{~B} \pm 0,416$ & $1,062 \mathrm{C} \pm 0,088$ \\
\hline \multicolumn{6}{|l|}{ Panela pressão } \\
\hline Microondas & $0,566 \mathrm{BC} \pm 0,048$ & $0,154 \mathrm{~B} \pm 0,056$ & $1,638 \mathrm{~B} \pm 0,049$ & $0,893 \mathrm{~B} \pm 0,281$ & $1,510 \mathrm{~B} \pm 0,102$ \\
\hline Valor de p & $<0,001$ & $<0,001$ & $<0,001$ & $<0,001$ & $<0,001$ \\
\hline \multicolumn{6}{|c|}{ Casca } \\
\hline Cru & $0,909 A \pm 0,028$ & $0,289 A \pm 0,081$ & $3,317 \mathrm{~A} \pm 0,355$ & $2,670 \mathrm{~A} \pm 0,243$ & $2,917 \mathrm{~A} \pm 0,144$ \\
\hline Imersão & $0,316 \mathrm{C} \pm 0,021$ & $0,111 \mathrm{~B} \pm 0,059$ & $1,536 C \pm 0,247$ & $0,474 \mathrm{~B} \pm 0,321$ & $1,178 \mathrm{C} \pm 0,179$ \\
\hline \multirow[t]{2}{*}{ Vapor } & $0,534 \mathrm{~B} \pm 0,044$ & $0,143 \mathrm{AB} \pm 0,035$ & $2,428 \mathrm{~B} \pm 0,500$ & $0,652 \mathrm{~B} \pm 0,017$ & $1,676 \mathrm{~B} \pm 0,079$ \\
\hline & $0,344 \mathrm{C} \pm 0,034$ & $0,141 \mathrm{AB} \pm 0,076$ & $1,410 \mathrm{C} \pm 0,348$ & $0,159 \mathrm{C} \pm 0,022$ & $1,130 \mathrm{C} \pm 0,172$ \\
\hline \multicolumn{6}{|l|}{ Panela pressão } \\
\hline Microondas & $0,575 \mathrm{~B} \pm 0,076$ & $0,196 \mathrm{AB} \pm 0,094$ & $2646 \mathrm{AB} \pm 0,328$ & $0,539 \mathrm{~B} \pm 0,0061$ & $1,504 \mathrm{~B} \pm 0,114$ \\
\hline Valor de $p$ & $<0,001$ & $<0,001$ & $<0,001$ & $<0,001$ & $<0,001$ \\
\hline
\end{tabular}

Médias seguidas pela mesma letra, na coluna, não diferem significativamente entre si, a 5\% de probabilidade, pelo teste de Tukey.

O tratamento em que a polpa de cenoura foi submetida ao cozimento com o uso do vapor manteve os maiores teores de proteínas, mantendo $57,75 \%$ do total de proteínas em relação ao vegetal cru. Já para casca da cenoura os tratamentos em que esta foi submetida ao cozimento com o uso do vapor e com o uso do microondas foram os que mantiveram os maiores teores de proteínas. O tratamento com o uso do microondas manteve $63,25 \%$ do total de proteínas em relação ao vegetal cru, e o tratamento com o uso vapor manteve $58,74 \%$.

Para os teores de lipídios na polpa de cenoura não houve diferença estatística significativa para os tratamentos em todos os métodos de cocção utilizados, no entanto, observou-se diferença em relação ao vegetal cru, ocorrendo perda média significativa de $52 \%$ em todos os tratamentos. Na casca da cenoura quando o tratamento utilizado foi o micro-ondas, manteve-se $67,8 \%$ do total de lipídios em relação ao vegetal cru, os tratamentos da panela de pressão e vapor mantiveram 48,79 e 49,48\% respectivamente.

Os teores de fibra para a polpa de cenoura variaram apenas em relação ao vegetal cru, sem diferença entre os tipos de cozimento. As amostras de polpa de cenoura mantiveram em média 45,83\% da fibra em relação ao vegetal cru. O tratamento em que as cascas foram submetidas ao cozimento no vapor manteve $73,19 \%$ do total de fibras, já o tratamento com o uso do micro-ondas manteve $79,77 \%$ quando comparadas com o vegetal cru.

Os teores de açúcares redutores na polpa de cenoura, não apresentaram diferença significativa entre os métodos de cocção utilizados, porém houve diferença entre a amostra crua e os diferentes tipos de tratamentos. Houve uma perda média de $74 \%$ do total de açúcares redutores quando comparados com o vegetal cru.
O tratamento cuja casca da cenoura foi submetida ao cozimento sob vapor e com o uso do micro-ondas apresentaram a menor perda do nutriente em relação aos demais tratamentos comparados ao vegetal cru, o tratamento usando o microondas manteve $20,18 \%$ do total de açúcares redutores e o tratamento usando o vapor manteve $24,40 \%$ do total de açúcares redutores quando comparados com o vegetal cru. Os teores de açúcares redutores totais não foram encontrados na casca da cenoura.

Na polpa da cenoura observou-se perdas de 48,24\% para os teores de ácido ascórbico no tratamento com o uso do microondas, em relação ao vegetal cru. Este foi o método de cozimento que resultou em menores perdas do ácido ascórbico. Na casca da cenoura, observou-se perdas de $42,55 \%$ para o tratamento a vapor, e de $4,21 \%$ para o tratamento com o uso do microondas em relação ao vegetal cru, sendo verificado então que os tratamentos mais efetivos na manutenção dos teores de ácido ascórbico foram aqueles em que a casca foi submetida ao cozimento no vapor e microondas.

Moraes et al. (2010) observaram grande redução de vitamina $C$ na cenoura após cocção, a mesma foi colocada para cozinhar antes da água entrar em ebulição e o tempo foi superior ao necessário, elevando as chances de perdas por lixiviação e pelo calor. Além disso, a cenoura foi colocada para cozinhar já picada, o que agrava a perda de vitaminas. Segundo Zhang e Hamauz (2004), a cocção provoca mudanças nas características físicas e na composição química dos vegetais, principalmente no teor de vitamina C, devido a sua alta solubilidade e instabilidade térmica. Nesse presente trabalho observouse também a grande perda de ácido ascórbico após os cozimentos na casca e na polpa da cenoura. 
Os minerais avaliados na polpa e casca são apresentados na Tabela 3. Observou-se que a casca da cenoura crua possui teores de ferro, fósforo, potássio, cálcio, magnésio e zinco superiores à polpa da cenoura. As porcentagens são respectivamente de 38,10\%, 95,12\%, 47,04\%,
58,88\%, 70,27\% e 21,27\% a mais em relação à polpa. Portanto, sugere-se o consumo integral das hortaliças como fontes de minerais.

Tabela 3 - Minerais (\%) para a polpa e casca da cenoura, submetidas a diferentes métodos de cocção.

\begin{tabular}{|c|c|c|c|c|c|c|}
\hline $\begin{array}{l}\text { Tipo de } \\
\text { cozimento }\end{array}$ & $\mathrm{Fe}(\%)$ & $P(\%)$ & $\bar{K}(\%)$ & $\mathrm{Ca}(\%)$ & $\operatorname{Mg}(\%)$ & $\mathrm{Zn}(\%)$ \\
\hline \multicolumn{7}{|c|}{ Polpa } \\
\hline Cru & $243,3 \mathrm{~A} \pm 40,4$ & $4,1 \mathrm{~A} \pm 0,1$ & $54,2 \mathrm{~A} \pm 4,3$ & $9,0 \mathrm{~A} \pm 1,0$ & $3,7 \mathrm{~A} \pm 0,6$ & $55,0 \mathrm{~A} \pm 10,0$ \\
\hline Imersão & $93,0 \mathrm{C} \pm 7,9$ & $3,0 \mathrm{~B} \pm 0,1$ & $34,1 \mathrm{~B} \pm 4,3$ & $4,8 \mathrm{BC} \pm 0,2$ & $1,85 \mathrm{~B} \pm 0,1$ & $35,7 \mathrm{~B} \pm 0,6$ \\
\hline Vapor & $60,3 \mathrm{C} \pm 12,7$ & $3,1 \mathrm{~B} \pm 0,2$ & $39,5 \mathrm{~B} \pm 1,3$ & $3,7 \mathrm{C} \pm 0,4$ & $1,5 \mathrm{~B} \pm 0,1$ & $39,3 \mathrm{~B} \pm 2,1$ \\
\hline $\begin{array}{l}\text { Panela } \\
\text { pressão }\end{array}$ & $145,3 \mathrm{~B} \pm 12,7$ & $3,1 \mathrm{~B} \pm 0,3$ & $54,0 \mathrm{~A} \pm 4,0$ & $6,0 \mathrm{~B} \pm 0,4$ & $2,1 \mathrm{~B} \pm 0,1$ & $34,0 \mathrm{~B} \pm 1,7$ \\
\hline Microondas & $86,0 \mathrm{C} \pm 8,9$ & $3,4 \mathrm{~B} \pm 0,9$ & $36,0 \mathrm{~B} \pm 6,0$ & $3,5 \mathrm{C} \pm 0,1$ & $1,4 \mathrm{~B} \pm 0,1$ & $38,0 \mathrm{~B} \pm 0,0$ \\
\hline Valor de p & $<0,001$ & $<0,001$ & $<0,001$ & $<0,001$ & $<0,001$ & $<0,001$ \\
\hline \multicolumn{7}{|c|}{ Casca } \\
\hline Cru & $336,0 \mathrm{~A} \pm 39,3$ & $80, A \pm 0,1$ & $79,7 \mathrm{~A} \pm 9,5$ & $14,3 \mathrm{~A} \pm 2,1$ & $6,3 \mathrm{~A} \pm 0,6$ & $66,7 \mathrm{~A} \pm 2,1$ \\
\hline Imersão & $179,3 \mathrm{~B} \pm 12,4$ & $3,5 \mathrm{C} \pm 0,1$ & $32,3 \mathrm{C} \pm 0,4$ & $7,8 \mathrm{~B} \pm 0,3$ & $2,0 \mathrm{~B} \pm 0,1$ & $34,3 \mathrm{BC} \pm 0,6$ \\
\hline Vapor & $145,3 \mathrm{~B} \pm 5,5$ & $4,4 \mathrm{~B} \pm 0,4$ & $53,48 \mathrm{~B} \pm 3,0$ & $56,2 \mathrm{~B} \pm 0,1$ & $2,1 \mathrm{~B} \pm 0,1$ & $34,0 \mathrm{BC} \pm 1,7$ \\
\hline $\begin{array}{l}\text { Panela } \\
\text { pressão }\end{array}$ & $207,7 \mathrm{AB} \pm 15,0$ & $3,1 C \pm 0,1$ & $31,6 \mathrm{C} \pm 5,7$ & $7,2 \mathrm{~B} \pm 2,7$ & $1,7 \mathrm{~B} \pm 0,1$ & $37,7 \mathrm{~B} \pm 1,2$ \\
\hline Microondas & $122,3 \mathrm{~B} \pm 0,6$ & $4,8 \mathrm{~B} \pm 0,1$ & $52,4 \mathrm{~B} \pm 1,7$ & $5,2 \mathrm{~B} \pm 0,1$ & $2,0 \mathrm{~B} \pm 0,1$ & $32,3 \mathrm{C} \pm 2,1$ \\
\hline Valor de p & $<0,001$ & $<0,001$ & $<0,001$ & $<0,001$ & $<0,001$ & $<0,001$ \\
\hline
\end{tabular}

Médias seguidas pela mesma letra, na coluna, não diferem significativamente entre si, a 5\% de probabilidade, pelo teste de Tukey.

Observou-se que o tratamento com o uso da panela de pressão na polpa e na casca de cenoura manteve os maiores teores de ferro em relação aos demais tratamentos. Este manteve respectivamente 59,72\% e 43,24 para polpa e casca da cenoura, do total de ferro comparando-o com a amostra crua.

Para os teores de fósforo na polpa de cenoura não houve diferença estatística significativa para os tratamentos em todos os métodos de cocção utilizados, porém houve diferença entre os tratamentos utilizados e o vegetal cru, houve uma perda média de 23,18\% em todos os tratamentos quando comparados ao vegetal cru. Na casca verificou-se que o tratamento a vapor e com o uso do micro-ondas obtiveram os maiores teores de fósforo em relação aos demais tratamentos. O uso do micro-ondas manteve 60 \% do total de fósforo em relação ao vegetal cru, e o tratamento com o uso do vapor manteve 55\% do total de potássio em relação ao vegetal cru.

O tratamento com o uso da panela de pressão na polpa de cenoura foi o que manteve os maiores teores de potássio. Portanto esse manteve 99,63\% do teor de potássio na polpa da cenoura em relação ao vegetal cru. Já na casca os tratamentos com o uso do vapor e com o uso do micro-ondas foram os que mantiveram maiores quantidades de potássio na casca da cenoura. O tratamento com o uso do vapor manteve $67 \%$ do teor de potássio em relação ao vegetal cru e com o uso do micro-ondas manteve $65,7 \%$.

Verificou-se que para os teores de cálcio na polpa de cenoura, que o tratamento utilizado mais efetivo foi com o uso da panela de pressão, que manteve $66,67 \%$ do total de cálcio comparado com a amostra crua. Na casca de cenoura verificou-se que não houve diferença estatística significante entre os tratamentos de cozimentos utilizados, no entanto, ocorreu diferença entre amostra crua e os diferentes tipos de tratamentos, onde se observou uma perda em média de $46,15 \%$ do total de cálcio quando comparados com a amostra crua.

Quanto aos teores de magnésio na polpa de cenoura, não houve diferença estatística entre os tratamentos utilizados, no entanto, houve diferença estatística entre a amostra crua e entre os tratamentos com os diferentes cozimentos, observou-se uma perda de 54\% em média entre os tratamentos utilizados, quando comparados com a amostra crua. Na casca da cenoura, também não houve diferença estatística significante entre os tratamentos de cozimentos utilizados, no entanto, ocorreu diferença entre a mostra crua e os diferentes tipos de tratamentos, onde se observou uma perda em média de 30,95\% do total de cálcio quando comparados com a amostra crua.

Em relação aos teores de zinco na polpa de cenoura, também não houve diferença estatística entre os 
tratamentos utilizados, no entanto, houve diferença estatística entre a amostra crua e os tratamentos com os diferentes cozimentos. Observou-se uma perda de $66,81 \%$ em média entre os tratamentos utilizados, quando comparados com a amostra crua. Na casca da cenoura o tratamento com o uso da panela de pressão foi o tratamento que manteve os maiores teores desse mineral, esse manteve $56,52 \%$ do total de zinco quando comparados com o vegetal cru.

Cuppari et al. (2004) observaram em estudo realizado em alguns alimentos (abóbora, cenoura, chuchu, batata, berinjela e beterraba) que após uma cocção a concentração dos minerais diminui significantemente em relação ao alimento cru. Os autores constataram que a perda percentual total de potássio na maioria dos alimentos testados ficou entre 80 e $91 \%$, somente dois deles entre 58 e $65 \%$ apresentando em média $79 \%$. Moreira (2006) relatou em seu trabalho que todos os minerais avaliados nas hortaliças estudadas tiveram seus teores diminuídos nos métodos de cocção na água e no vapor. Na presente pesquisa o método de cozimento à vapor e em microondas resultaram nas menores perdas nutricionais na polpa e casca de cenoura, provavelmente devido o fato das amostras não entrarem em contato direto com à água no momento do cozimento.

\section{CONCLUSÃO}

No vegetal cru, a casca da cenoura apresentou composição nutricional equivalente à polpa para proteína, lipídios, fibra, açúcares redutores e totais e ácido ascórbico. Já os teores de minerais foram superiores na casca em relação à polpa. Ocorreram perdas de nutriente na polpa e casca de cenoura submetida aos diferentes métodos de cozimento em relação ao vegetal cru. O método de cozimento à vapor e em microondas resultaram nas menores perdas nutricionais na polpa e casca de cenoura.

\section{REFERÊNCIAS}

\section{ASSOCIATION OF OFFICIAL ANALYTICAL}

CHEMISTS. Official methods of analysis. Washington: Association of Official Agricultural Chemists, p. 937, 1984.

AGROOLHAR.Cenoura é a $5^{\circ}$ hortaliça mais cultivada no Brasil e produção anual chega a 25 mil toneladas. 2013. Disponivel em:

,http://www.olhardireto.com.br/agro/noticias/exibir.asp? noticia=Cenoura_e_o_5_alimento_mais_cultivado_no_B rasil_e_producao_anual_chega_a_25_mil_toneladas\&edt $=9 \& \overline{\mathrm{id}}=10603$

CAMPOS, M. F., LIMA, S. A., PASQUI, C. S., MAIA, G. E. G., Determinação dos Teores de Vitamina c em Hortaliças Minimamente Processadas. Revista

Alimentos e Nutrição, Araraquara, v.19, n.3, p.329-335, jul/ set.2008.
CECCHI, H.M. Fundamentos teóricos e práticos em análise de alimentos. Campinas: UNICAMP, p. 211, 1999.

COMBS JR, G. F. The vitamins: fundamental aspects in nutrition and health. 2nd ed. New York: Academic, 1998. $526 \mathrm{p}$.

CUPPARI, et al. Preparo de vegetais para utilização em dieta restrita em potássio.Nutrire: Revista da Sociedade Brasileira de Alimentos e Nutrição, São Paulo, SP, v.28, p.1-7, dez.2004.

FRANCO, G. Tabela de composição química dos alimentos. 9.ed. São Paulo: Atheneu, 2004. 307p.

GOKOGLU, N.; YERLIKAYA, P.; CENGIZ, E. Effects of cooking methods on the proximate composition and mineral contents of rainbow trout (Oncorhynchus mykiss). Food Chemistry, London, v. 84, n. 1, p. 19-22, Jan. 2004.

LANA, M.M. Aspectos da fisiologia de cenoura minimamente processada. Horticultura Brasileira, Brasília, v. 18, n. 3, p. 154-158, 2000.

LEE, S.K.; KADER, A.A. Preharvest and postharvest factors influencing vitamin $C$ content of horticultural crops. Postharvest Biology and Technology, Amsterdam, 20, p. 207-220, 2000.

LIMA, K. S. C; LIMA, A. L. S; FREITAS, L. C; DELLA-MODESTA, R. C; GODOY, R. L. Efeito de baixas doses de irradiação nos carotenóides majoritários em cenouras prontas para o consumo. Ciência e Tecnologia de Alimentos, Campinas, v. 24, n. 2,, p. 183-193, 2004.

MALAVOLTA, E.; VITTI, G. C.; OLIVEIRA, S.A. Avaliação do estado nutricional de plantas: princípios e aplicações. Piracicaba, p. 201, 1989.

MAPA. Ministério da Agricultura, Pecuária e Abastecimento. Manual de Métodos de Análises de Bebidas e Vinagre. Acesso em 11/02/2008. Disponível em

http://www.agricultura.gov.br/portal/page?_pageid=33,1 041355\&_dad=portal\&_schema=PORTAL.

MONTEIRO, A. B. Valor Nutricional de partes Convencionais e Não Convencionais de Frutas e Hortaliças.p.62. 2009. Dissertação apresentada à faculdade de Ciências Agronômicas da Unesp-Campus de Botucatu, para obtenção do título de Mestre em Agronomia (Energia na Agricultura), Nov/ Botucatu SP.

MORAES, F.A., COTA, A.M., CAMPOS, F.M., PINHEIRO-SANT'ANA, M. Perdas de vitamina C em hortaliças durante o armazenamento, preparo e distribuição em restaurantes. Ciência e Saúde Coletiva, Rio de Janeiro, v. 15, n.1, p.51-62, 2010. 
MOREIRA, R. T. Análise de perdas de mineiras em hortaliças submetidas a dois métodos de cocção. 33p. 2006.Trabalho Final de Graduação apresentado ao Curso de Nutrição- Área de Ciências da Saúde, do Centro Universitáio Franciscano, como requisito parcial para obtenção do grau de nutricionista-Bacharel em Nutrição.,CentroUniversitário São Francisco-Unifra.

NELSON, N. A. Pthotometric adaptation of the Somogy method for the determination of glucose. Journal of Biological Chemistry, Baltimore, n. 153, p. 375-80, 1944.

RAMIREZ-CARDENAS, L.; LEONEL, A. J.; COSTA, N. M. B.. Efeito do processamento doméstico sobre o teor de nutrientes e de fatores antinutricionais de diferentes cultivares de feijão comum. Ciência e Tecnologia de Alimentos, Campinas, v. 28, n. 1, p.200-213, 2008 .

RIOS, M. D. G.; PENTEADO, M. V. C. Vitamina 36. C. In: PENTEADO, M. V. C. Vitaminas: aspectos nutricionais, bioquímicos, clínicos e analíticos. São Paulo: Manole, 2003. p 201-225.

SANTOS, M. A. T.; ABREU, C. M. P.; CARVALHO, V. D. Efeito de diferentes tempos de cozimento nos teores de minerais em folhas de brócolis, couve-flor e couve. Ciência e Agrotecnologia, Lavras. V.27, n.3, p.597-604, 2003.

SOMOGY, M. Determination of blood sugar. Journal of Biological Chemistry, Baltimore, n. 160, p. 69-73, 1945.

SPAGNOL, W. A.; PARK, K. J.; SIGRIST, J. M. M. Taxa de respiração de cenouras minimamente processadas e armazenadas em diferentes temperaturas. Ciência e Tecnologia de Alimentos, Campinas, v. 26, n. 3, p. 550-554, 2006.

STATISTICAL ANALYSIS SYSTEM - SAS. SAS/STAT User's Guide. version 6.12. 4.ed, v.2, Cary: 2003. 842p.

WATADA, A.E., QI, L. Quality of fresh-cut produce. Postharvest Biology and Technology, Amsterdam, v.15, p.201-205, 1999.

ZADERNOWSKI, R.; BOROWSKA, J.; KOWALSA, M.; BUDREWICZ, G.; SZAJDEK, A., Quality of carrots juice as conditioned by raw material and technology, Science Research, p.183-192, 2003.

ZHANG, D; HAMAUZU, Y. Phenolics, ascorbic acid, carotenoids and antioxidant activity of broccoli and their chances during convencional and microwave cooking. Food Chemistry, London, v. 88, p. 503-509, 2004. 